\title{
Bilateral nanophthalmos, pigmentary retinal dystrophy, and angle closure glaucoma-a new syndrome?
}

\author{
SUPRIYO GHOSE, MAHIPAL S SACHDEV, AND HARSH KUMAR \\ From the Dr Rajendra Prasad Centre for Ophthalmic Sciences, All-India Institute of Medical Sciences, Ansari \\ Nagar, New Delhi - 110029, India
}

SUMmARY An unusual case of bilateral nanophthalmos with pigmentary retinal dystrophy and angle closure glaucoma is presented. This is probably the first published report of the established association of all these three entities in the same patient. The aetiological possibilities and clinical significance are discussed.

The not infrequent association of angle closure glaucoma with primary pigmentary degeneration of the retina has been known since $1862 . .^{\prime}$ The susceptibility of microphthalmic eyes to develop angle closure glaucoma is well established. ${ }^{2} \mathrm{~A}$ few reports are also available of microphthalmos with pigmentary retinal dystrophy. ${ }^{3}$ Interestingly enough, the manifestation of all these three disorders in the same patient does not seem to have been conclusively documented previously.

\section{Case report}

A 56-year-old male teacher presented complaining of poor vision, more so in the right than the left eye, since childhood. He had used high hypermetropic glasses since the age of 12 years, when he had a corrected vision of $6 / 12$ in both eyes. $\mathrm{He}$ also complained of night blindness associated with progressively worsening vision since the age of 30 . Over the last 10 years the vision had further deteriorated in his right eye to finger counting only. Transient blurring of vision on near work had been present for the past six months, without any associated pain or watering.

He was given elsewhere a course of subconjunctival injections in both eyes, but without improvement. However, the vision in the left eye remained useful. No history of pain, redness, watering, or

Correspondence to Dr Supriyo Ghose, Dr Rajendra Prasad Centre for Ophthalmic Sciences, All-India Institute of Medical Sciences, Ansari Nagar, New Delhi - 110029, India. coloured haloes could be elicited. His personal and family histories were non-contributory.

General examination revealed a tall, averagely built male, with no systemic abnormality. On ocular examination, his best corrected visual acuity was counting fingers at 2 metres OD and 6/24 OS with +14 Dsph OU. The eyeballs were deep set (Fig. 1) with narrowed palpebral apertures, small corneas (horizontal diameter of $9.5 \mathrm{~mm}$ both eyes), markedly shallow anterior chambers, and minimal lenticular changes. The pupils showed an anisocoria; the right pupil was the larger (Figs. 2a, b) and gave a poor

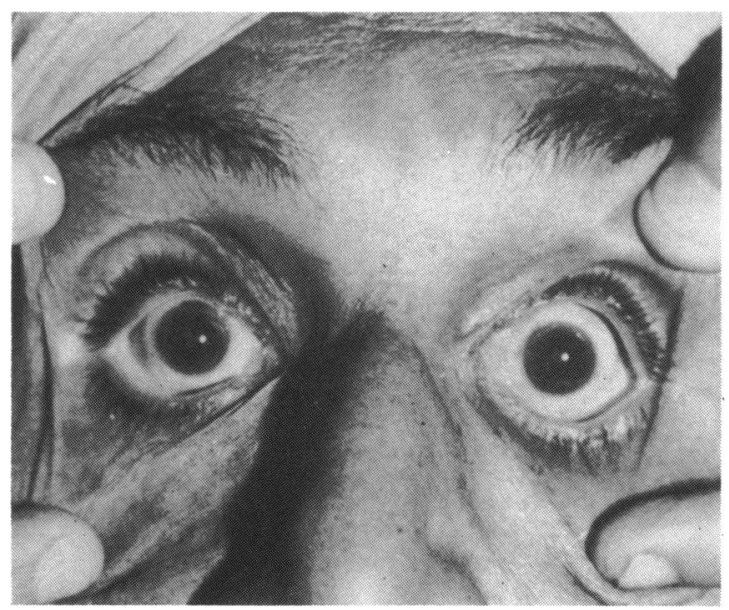

Fig. 1 Note the deep set small eyeballs. 


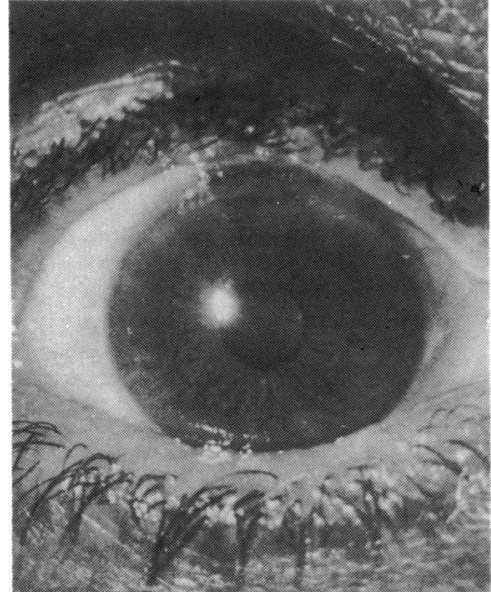

Fig. $2 a$

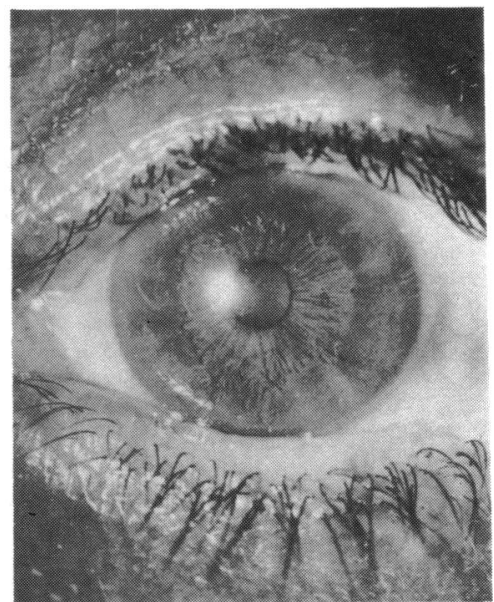

Fig. $2 b$
Fig. 2 Close-ups of the (a) right eye and (b) left eye. Note the larger pupil in the right eye, with a normal iris pattern. direct response to light. The consensual light reflex in the left eye was also sluggish. No patch of iris atrophy was noted in either eye.

Ophthalmoscopic examination through slightly hazy media revealed hypermetropic discs with mild pallor (more marked in the right eye), normal cupdisc ratios, attenuated arteries, and bone spicule pigmentation together with clumps of pigment, especially in the perivascular area in the equatorial region (Fig. 3). The macula also showed pigmentation, more in the right than the left eye.

On biomicroscopy only a chink of the anterior chamber about $1 / 4$ the thickness of the cornea was found adjacent to the limbus in both the eyes (Figs. $4 a, b, 5 a, b)$.

On investigation the intraocular pressures (IOP)

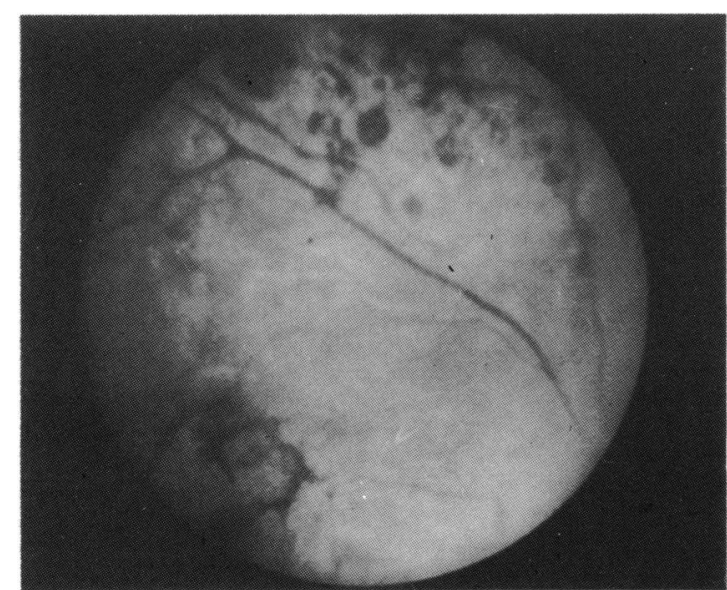

Fig. 3 Right eye fundus photograph (through slightly hazy media) showing a pale disc, attenuated arteries, and pigmentary dystrophy. Note the macular involvement. were normal. A narrow angle with markedly narrow entry was observed gonioscopically in both eyes. Dry refraction failed to improve the vision any further. On Goldmann perimetry, a bilateral constriction of both central and peripheral fields, with a ring scotoma in the $20-30^{\circ}$ isoptres, was recorded.

Anterior chamber depths of $1.90 \mathrm{~mm}$ OD and 1.94 $\mathrm{mm}$ OS were measured by the Haag-Streit 900 pachometer. On A-scan biometry the axial lengths were $18.2 \mathrm{~mm}$ OD and $18.3 \mathrm{~mm}$ OS, with a lens thickness of $4.9 \mathrm{~mm} \mathrm{OU}$, and anterior chamber depths of $1.90 \mathrm{~mm}$ OD and $1.92 \mathrm{~mm}$ OS.

Electroretinography recordings (without pupillary dilatation) were subnormal in both eyes. Visually evoked responses using the pattern reversal stimuli showed no detectable pattern in the right eye and an amplitude of $8 \mu \mathrm{V}$ with a latency of $105 \mathrm{~ms}$ in the left eye (control amplitude 6-10 $\mu \mathrm{V}$; latency $<100 \mathrm{~ms}$ ).

The visual prognosis was explained to the patient. In view of his shallow anterior chambers and high hypermetropic status he was not advised to have provocative tests or miotics, but only a periodic follow-up with us, for which he regularly attended for four months.

Six months later he presented with complaints of nausea, blurred vision, coloured haloes, redness, and pain and watering of four days duration in the right eye. Owing to unavoidable circumstances he could not attend the hospital immediately on the onset of these symptoms, though he had been told of the possibility of their occurrence and their significance.

Ocular examination carried out then revealed a best corrected visual acuity of finger counting at 1 metre OD and 6/24 OS. Other salient features in the right eye included marked conjunctival and ciliary congestion, corneal oedema, a few keratic precipitates with an aqueous flare, a somewhat oval, fixed, 
Fig. 4 Initial slit-lamp photographs of the right eye. Note the (a) markedly reduced depth of the anterior chamber adjacent to the limbus, more evident in (b) $a$ peripheral section.

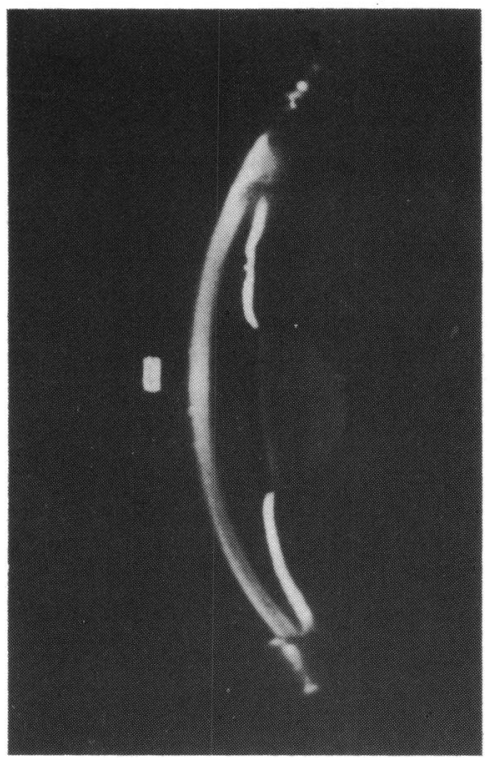

Fig. $4 \mathrm{a}$

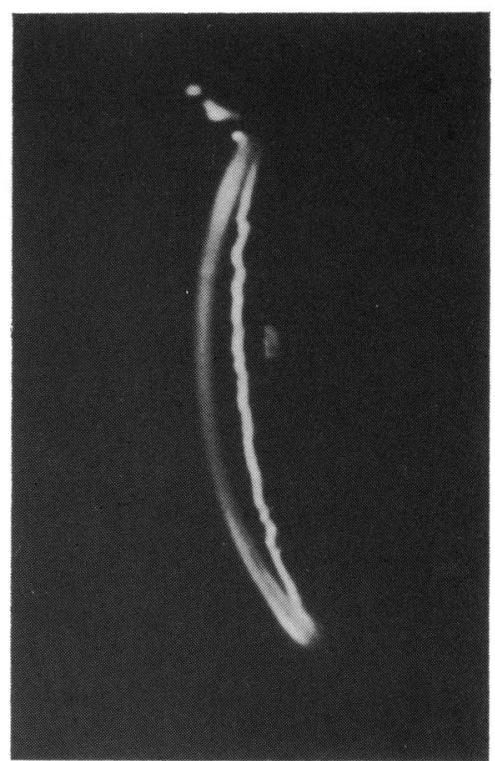

Fig. 4b mid-dilated pupil with sphincteric atrophy (Figs. 6a, b), and a raised IOP of $54 \mathrm{mmHg}$ in the right eye. On gonioscopy a closed angle with peripheral anterior synechiae in the right eye and a narrow angle with narrow entry as before in the left eye were observed. A diagnosis of an acute attack of angle closure glaucoma in the right eye was made and medical therapy promptly instituted. The control of IOP in the right eye being inadequate on miotics alone, a trabeculectomy was performed, resulting in satisfactory control of the IOP postoperatively. $\mathrm{He}$ is awaiting a prophylactic peripheral iridectomy in the left eye.

\section{Discussion}

The term nanophthalmos (pure microphthalmos) indicates an eye which is normal other than being
Fig. $5(\mathrm{a}, \mathrm{b})$ Slit-lamp photographs of the left eye showing features similar to those of the right eye (as seen in Fig. 4).

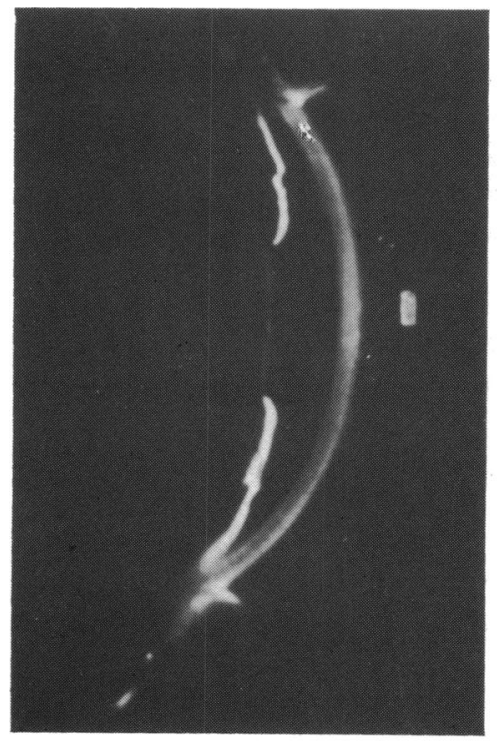

Fig 5a

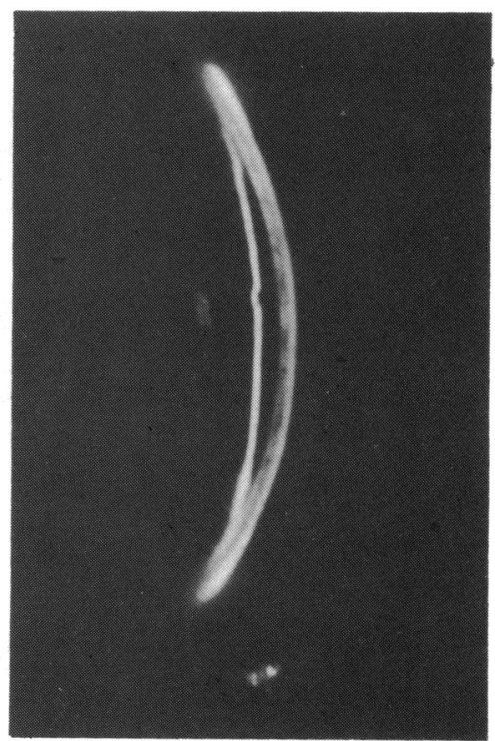

Fig. 5b 


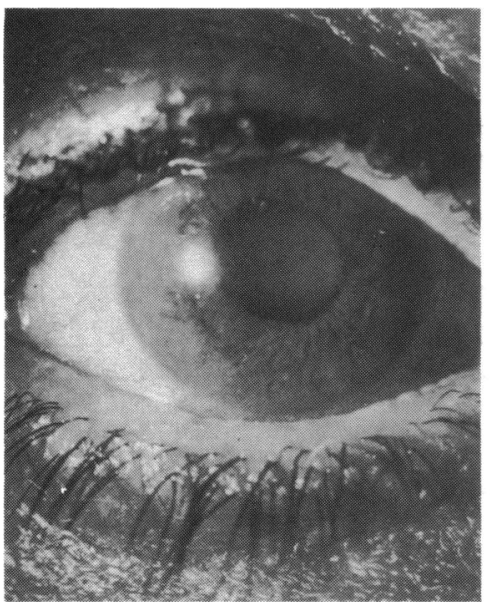

Fig. 6a

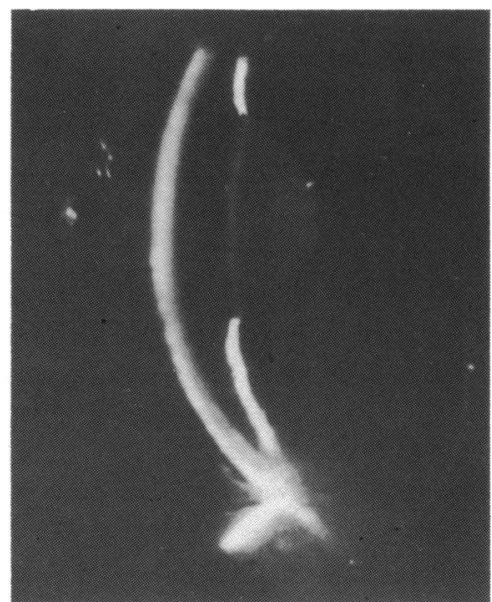

Fig. 6b

Fig. 6 (a) Close-up of the right eye during acute attack of angle closure glaucoma: the hazy cornea and oval mid-dilated pupil with sphincteric atrophy are obvious. (b) The corneal oedema is more evident in the slit-lamp section: the peripheral chink of anterior chamber is no longer visible (compare with Fig. 4a).

uniformly reduced in size. ${ }^{245}$ Measurements of the nanophthalmic eye have been infrequently documented. The critical value of the eyeball dimensions at which an eye is judged nanophthalmic have not been clearly defined. Two-thirds of the normal volume and an axial length of $16-18.5 \mathrm{~mm}$ have been proposed. ${ }^{4} \mathrm{~A}$ reduction of the normal eye size by $15 \%$ has also been suggested as a sufficient criterion for diagnosing nanophthalmos. ${ }^{5}$ By these criteria our patient has bilateral nanophthalmos. However, a nanophthalmic eye has also been categorised as one having a sagittal diameter of $13-17 \mathrm{~mm} .{ }^{6}$ Besides congenital angle anomalies, which are common ${ }^{4}$ and predispose nanophthalmic eyes to simple glaucoma later, these eyes are usually hypermetropic, with a relatively large lens and a remarkably high ratio of lens volume to eye volume. ${ }^{7}$ This plays an important contributory role in the shallow anterior chamber encountered in nanophthalmos. Presumably growth of the lens with age increasingly crowds the already developmentally shallow anterior chamber, thereby rendering these eyes susceptible to angle closure glaucoma, as in our case.

Our findings on investigation of the patient corroborated the clinical diagnosis of pigmentary retinal dystrophy. The clumps of pigment along with the classical bone spicule pigmentation, together with the macular involvement, are recognised variant features. Primary pigmentary degeneration of the retina is well known in having certain systemic and ocular associations, of which glaucoma is well documented. Most authors mention open angle glaucoma. ${ }^{8}$ The association of angle closure glaucoma with retinitis pigmentosa seems to be rather rare in published reports, and it has even been proposed that this association may be fortuitous." Whatever the type of glaucoma, the clinical and histopathological reports available have not elucidated its pathogenesis in relation to retinitis pigmentosa. ${ }^{19}$

Maldevelopment of the retina, retinitis pigmentosa, and other retinal anomalies have also been reported in nanophthalmos. ${ }^{34}$ The aetiology of the association of nanophthalmos and retinitis pigmentosa also remains unexplained. It is possible that in the basically underdeveloped eye of nanophthalmos the lack of full development of the retina in the later part of fetal life is responsible for pigmentary retinal dystrophic changes.

The association of nanophthalmos with pigmentary retinal dystrophy, ${ }^{3}$ pigmentary retinal dystrophy with glaucoma, ${ }^{189}$ and glaucoma with nanophthal$\operatorname{mos}^{24-7}$ has been reported previously. However, the simultaneous occurrence of all these three entities, namely, nanophthalmos, pigmentary retinal dystrophy, and glaucoma, seems to have been described only twice. ${ }^{10}$ "The first report, ${ }^{10}$ in three brothers, was apparently of simple glaucoma, there being no mention of detailed symptomatology or of angle closure glaucoma. In the second report four patients of the 13 affected seem to have had ocular features suggestive of angle closure glaucoma, though no measurements of anterior chamber depth or gonioscopic or biometric data were included." Again, in this rather detailed communication there is no categorical mention of the term angle closure glaucoma. This report has subsequently been interpreted as 'microphthalmia' with a 'somewhat atypical dystrophia retinae pigmentosa' and 'ocular hypertension with subacute attacks'. ${ }^{3}$

In our patient there is no doubt of the established diagnosis of angle closure glaucoma in association with nanophthalmos and pigmentary retinal dystrophy. In the absence of other family members 
being affected this case seems to have been sporadic in nature.

Since nanophthalmic eyes seem to be anatomically predisposed to angle closure glaucoma, and pigmentary retinal dystrophy has been reported with both these entities, it is possible that the occurrence of all three in a given eye may have been overlooked in earlier reports. It appears that if a case presents with any two of these three entities, it is worth diligently searching for the third component, even if only to exclude it.

\section{References}

1 Gartner S, Schlossman A. Retinitis pigmentosa associated with glaucoma. Am J Ophthalmol 1949; 32: 1337-50.

2 Phelps CD. Glaucoma associated with retinal disorders. In: Ritch R, Shield MB, eds. The secondary glaucomas. St Louis: Mosby, 1982: 150-61.
3 Waardenburg PJ. In: Waardenburg PJ, Franceschetti A, Klein D, eds. Genetics and ophthalmology. Assen: Royal Van Gorcum, 1961; 1: 764.

4 Duke-Elder S. System of ophthalmology. Vol 3 Part 2. London: Kimpton, 1964; 3 (2): 488-95.

5 O'Grady RB. Nanophthalmos. Am J Ophthalmol 1971; 71: 1251-3.

6 Friedman AH, Kwitko ML, Ritch R. Glaucoma associated with congenital disorders. In: Ritch R, Shield MB, eds. The secondary glaucomas. St Louis: Mosby, 1982: 51-66.

7 Kimbrough RL, Trempe CS, Brockhurst RJ, Simmons RJ. Angle closure glaucoma in nanophthalmos. Am J Ophthalmol 1979; 88: 572-9.

8 Kogbe OI, Follmann P. Investigations into the aqueous humour dynamics in primary pigmentary degeneration of the retina. Ophthalmologica 1975; 171 : 165-75.

9 Duke-Elder S, Jay B. System of ophthalmology. London: Kimpton, 1969; 11: 692.

10 Walsh FB, Hoyt WF. Clinical neuroophthalmology. Baltimore: Williams and Wilkins, 1969; 1: 651-2.

11 Hermann P. Le syndrome microphtalmie-rétinite pigmentaireglaucome. Arch Ophthalmol (Paris) 1958; 18: 17-24. 